\title{
CURSOS DE EXTENSÃO EM ZOOLOGIA E SUAS IMPLICAÇÕES NO AMBIENTE ACADÊMICO
}

\author{
Thiago Mateus Rocha Santos \\ Universidade Federal de Mato Grosso do Sul \\ thiagomrsantos@gmail.com
}

\author{
Thales D. Leandro \\ Universidade Estadual Paulista \\ thaleshdias@gmail.com \\ Marcelo Oscar Bordignon \\ Universidade Federal de Mato Grosso do Sul \\ marcelo.bordignon@ufms.br
}

\begin{abstract}
Resumo
A zoologia é a ciência que estuda os animais e devido a suas diversas subáreas de estudo o direcionamento dos estudantes e o acesso ao conhecimento científico de qualidade geralmente torna-se limitado. Embora o mercado de trabalho possua demanda por profissionais qualificados, algumas peculiaridades técnicas, éticas e burocráticas envolvem o estudo com animais. Buscando fornecer ferramentas para atenuar carências na formação profissional, o Laboratório de Zoologia da Universidade Federal de Mato Grosso do Sul realiza anualmente cursos de extensão técnico-científicos em Zoologia. O primeiro curso ocorreu em 2009e foi intitulado como "Curso teórico-prático de taxidermização básica de mamíferos"e em virtude da sua demanda e aceitação foi realizado um segundo curso, o qual englobou conceitos de diversas subáreas da Zoologia focando as Coleções Zoológicas e foi intitulado como “Técnicas de Manejo de Coleções Zoológicas”. Os bons indicadores dos cursos iniciais levaram o projeto a mais quatro edições, sendo a última edição realizada em 2014. Os dados aqui relatados mostram bom aproveitamento pela comunidade acadêmica, contribuindo para a formação profissional de alunos, professores e da comunidade externa.
\end{abstract}

Palavras-chave: Animais. Coleções Zoológicas. Taxidermia.

\section{ZOOLOGY EXTENSION-PROGRAM PROJECTS AND THEIR IMPLICATIONS WITHIN ACADEMIC COMMUNITY}

\begin{abstract}
Zoology is the science that deals with animals and due to its number of sub areas of study, the academic orientation of students and the access to quality knowledge usually become restricted. Although the labour market has demanded by qualified professionals, many peculiarities involve the study with animals, such as techniques, ethics and bureaucracies. Aiming to provide tools to attenuate deficiencies from the academic formation of these professionals, the Laboratory of Zoology of the Universidade Federal de MatoGrosso do Sul (UFMS) annually realizes extension-program projects. The firstprojectwas in 2009andwasnamed as curso teórico-prático de taxidermização básica de mamíferos. Due its demand and receptivity for a great amount of people, a second project was offered comprising some concepts of several Zoology sub areas and Zoological Collections and was named as técnicas de Manejo de ColeçõesZoológicas. Good indicators from the early projects allowed four more editions, the last one in 2014. The projects were put to good use by the academic community, contributing for vocational training of students, professors, and external community.
\end{abstract}

Keywords: Animal. Zoological Collections. Taxidermy.

\section{CURSOS DE EXTENSIÓN EN ZOOLOGÍA Y SUS IMPLICACIONES EN LA COMUNIDAD ACADÉMICA}

Resumen

La Zoología es la ciencia que estudia los animales y debido a sus diferentes sub-áreas, es limitada la orientación a estudiantes y el acceso al conocimiento científico de calidad. Aunque el mercado de trabajo posee demanda por profesionales cualificados, algunas peculiaridades técnicas, éticas y burocráticas envuelven el estudio con animales. Con el objetivo de proporcionar herramientas para atenuar las deficiencias en la formación de profesionales, cada año el Laboratorio de Zoología de la Universidad Federal de Mato Grosso do Sul (UFMS) realiza cursos de extensión. El primer 
curso fue en 2009 y se titula "Curso teórico-práctico de taxidermización básica de mamíferos" y debido a su demanda y percepción, se proporcionó un segundo curso que involucró conceptos de varias sub áreas de la Zoología y Colecciones Zoológicas y se titula "Técnicas de manejo de colecciones zoológicas". Los buenos indicadores de los primeros cursos llevaron el proyecto a otras cuatro ediciones, la última en 2014 Los datos demuestran la contribución positiva de la comunidad académica, en la formación profesional de estudiantes, profesores y a la comunidad externa.

Palavras clave: Animal. Colecciones Zoológicas. Taxidermia. 
Cursos de extensão em zoologia e suas implicações no ambiente acadêmico

\section{INTRODUÇÃO}

A zoologia é a ciência que estuda os animais, grupo biológico com alta representatividade, englobando aproximadamente 1,3 milhão de espécies (BRUSCA E BRUSCA, 2007). Devido à sua elevada diversidade de espécies e tipos morfológicos epor ser fonte de recurso alimentar, os animais são objeto da nossa atenção e estão intimamente integrados à história evolutiva da humanidade pelo processo de caça e/ou domesticação (DIAMOND, 2002; VIGNE, 2011). De modo geral, documentários e reportagens sobre meio ambiente têm como foco principal a vida animal, uma vez que a temática desperta atenção e curiosidade na maioria do público.

Apesar da grande demanda de interesse pela área, o empenho de professores do ensino básico na abordagem de assuntos relacionados à Zoologia ainda é insatisfatório (GUIMARÃES, 2004). Nesta etapa do sistema educativo, o ensino de Zoologia baseia-se exclusivamente em abordagens teóricas, causando na maioria dos casos, desinteresse por parte dos alunos. Somado a isso, os poucos professores que aplicam outros recursos didáticos o fazem sem estudos prévios que avaliem sua eficácia (BORGES, 2000).

A fim de diminuir a deficiência na formação básica e dos profissionais que nela atuam, algumas Instituições de Ensino Superior (IES) desenvolvem ações em escolas da rede pública e privada (e.g., MARQUES, 2011), buscando identificar e sanar as necessidades dos alunos e professores por meio de palestras, oficinas e exposições. Além disso, a melhoria da qualidade do ensino de Zoologia pode contribuir para que alunos do ensino básico e superior direcionem suas curiosidades e desenvolvam interesse por esta ciência, embasando a tomada de decisão quando optam em seguir e se especializar nesta área.

No que se refere à produção científica, apenas o conhecimento em Zoologia não é suficiente para desenvolver bons trabalhos, uma vez que existem diversas questões metodológicas e burocráticas quando se trata de estudos com animais, especialmente quando envolve manipulação, criação, experimentação e/ou morte (GOLDENBERG, 2000; SCHNAIDER \& SOUZA, 2003). Este é um ponto importante de abordagem para profissionais em formação, contudo, devido à elevada carga horária dos cursos de graduação, a temática é geralmente negligenciada durante a elaboração dos projetos pedagógicos de curso.

Diante do panorama exposto, a partir de 2009 a equipe do Laboratório de Zoologia, do Centro de Ciências Biológicas e da Saúde (CCBS), da Universidade Federal de Mato Grosso do Sul (UFMS), realiza cursos de extensão sem fins lucrativos com o objetivo de aproximar a Zoologia e os métodos de estudo aos futuros profissionais e à comunidade externa. 
Cursos de extensão em zoologia e suas implicações no ambiente acadêmico

Considerando a importância da Zoologia como ciência, sobretudo das ações de extensão para o desenvolvimento da sociedade, o objetivo deste relato é divulgar os cursos de extensão em Zoologia realizados pela Universidade Federal de Mato Grosso do Sul, a fim de evidenciar sua potencialidade para construção de novos profissionais.

\section{MATERIAL E MÉTODOS}

Todos os cursos foram realizados seguindo as normas e diretrizes vigentes para elaboração de projetos de extensão da Universidade Federal de Mato Grosso Sul. Cada processo consistiu na elaboração e submissão de uma proposta na plataforma SIGPROJ (Sistema de Informação e Gestão de Projetos; http://sigproj1.mec.gov.br). Após a avaliação e aprovação pelo colegiado da unidade responsável, os projetos foram executados de acordo com o cronograma estabelecido.

Até o momento foram realizados seis cursos, sempre buscando atender a demanda de alunos e professores a cada nova edição. Aulas teóricas e práticas foram intercaladas para melhorar o aproveitamento dos assuntos abordados. Apresentações por meio de projeção foram utilizadas, uma vez que ferramentas modernas são tendências comprovadamente eficazes para a educação (MORAN et al., 2006; RAMOS \& CARNEIRO, 2009).

Os animais utilizados no desenvolvimento das aulas práticas foram provenientes de projetos de pesquisa de alunos dos Programas de Pós-Graduação da própria Instituição, devidamente mortos, com autorização do Comitê de Ética Institucional e IBAMA. Consistiram de roedores, preferencialmente não nativos (rato doméstico),ou sem interesse primordial para a Coleção da Instituição, uma vez que seriam utilizados para treinamento e poderiam tornar-se impróprios para coleções de referência.

Os materiais de consumo utilizados durante o curso (e.g., lâminas de bisturi, conservantes, algodão, etc.) foram adquiridos em comum acordo pelos próprios participantes.O material remanescente foi doado para o Laboratório de Zoologia da universidade para uso geral. 
Cursos de extensão em zoologia e suas implicações no ambiente acadêmico

\section{RESULTADOS E ANÁLISE}

A primeira proposta "Curso Teórico-Prático de Taxidermia Básica de Mamíferos" ocorreu em 2009 e teve carga horária de oito horas (Figura 1A). A abordagem inicial foi simples e consistiu no ensino de técnicas detaxidermia (empalhamento), procedimento rotineiro para trabalhos com determinados grupos animais que são incorporados em coleções de referência como aves e pequenos mamíferos (PALAUS, 1977). Naquele ano foram disponibilizadas dez vagas, as quais tiveram como público alvo estudantes de Ciências Biológicas da Universidade Federal de Mato Grosso do Sul, além da comunidade externa à Instituição. Como a demanda foi maior que o número de vagas disponibilizadas (10 vagas), a Comissão Organizadora optou por aceitar 15 participantes. A frequência mínima de participação foi de $75 \%$, porém todos os participantes obtiveram 100\% de frequência e executaram todas as atividades propostas.

As atividades do curso consistiram de duas partes: teórica e prática, totalizando quatro horas/aula cada. Na primeira parte foi abordada a história da taxidermia, utilizações atuais e diretrizes teóricas para a realização do procedimento. A segunda parte consistiu numa abordagem prática, onde os alunos se organizaram em duplas e com o auxílio de monitores aplicaram os conhecimentos teóricos na taxidermia de um animal.

Como normas do projeto cadastrado, a atividade passou por avaliação pelos participantes por meio de questionário com perguntas relacionadas às atividades teóricas e práticas. Das 15 avaliações, duas avaliaram o curso como "bom" e 13 como "ótimo". Dentre as sugestões, a principal e recorrente foi o aumento do número de vagas. A avaliação do curso nos permitiu concluir que a proposta foi satisfatória e atingiu de forma esperada o público alvo, contemplando as expectativas de ambas as partes. Tais resultados foram primordiais e subsidiaram a elaboração e execução das ações de extensão propostas nos anos seguintes.

A segunda ação de extensão “Técnicas de Manejo de Coleções Zoológicas” ocorreu em 2010 e teve como foco principal a gestão de coleções didáticas e científicas de Zoologia. Este curso foi essencialmente voltado para a capacitação profissional e sua carga horária totalizou 40 horas. A organização das aulas seguiu a estrutura da primeira proposta: aula prática seguida de aula teórica, desta vez englobando diversas abordagens referentes às coleções zoológicas, as quais foram divididas em três etapas. Inicialmente foi apresentado o conceito de coleção zoológica, diferenças entre coleções didáticas e científicas, funcionamento de coleções, tipos de coleções quanto ao armazenamento dos animais, sistemas de informação, legislações que dispõem sobre o 
funcionamento de coleções, bem como o histórico de acidentes e como evitá-los. A taxidermia foi abordada na segunda etapa do curso por meio atividades teórico-práticas (Figura 1B).
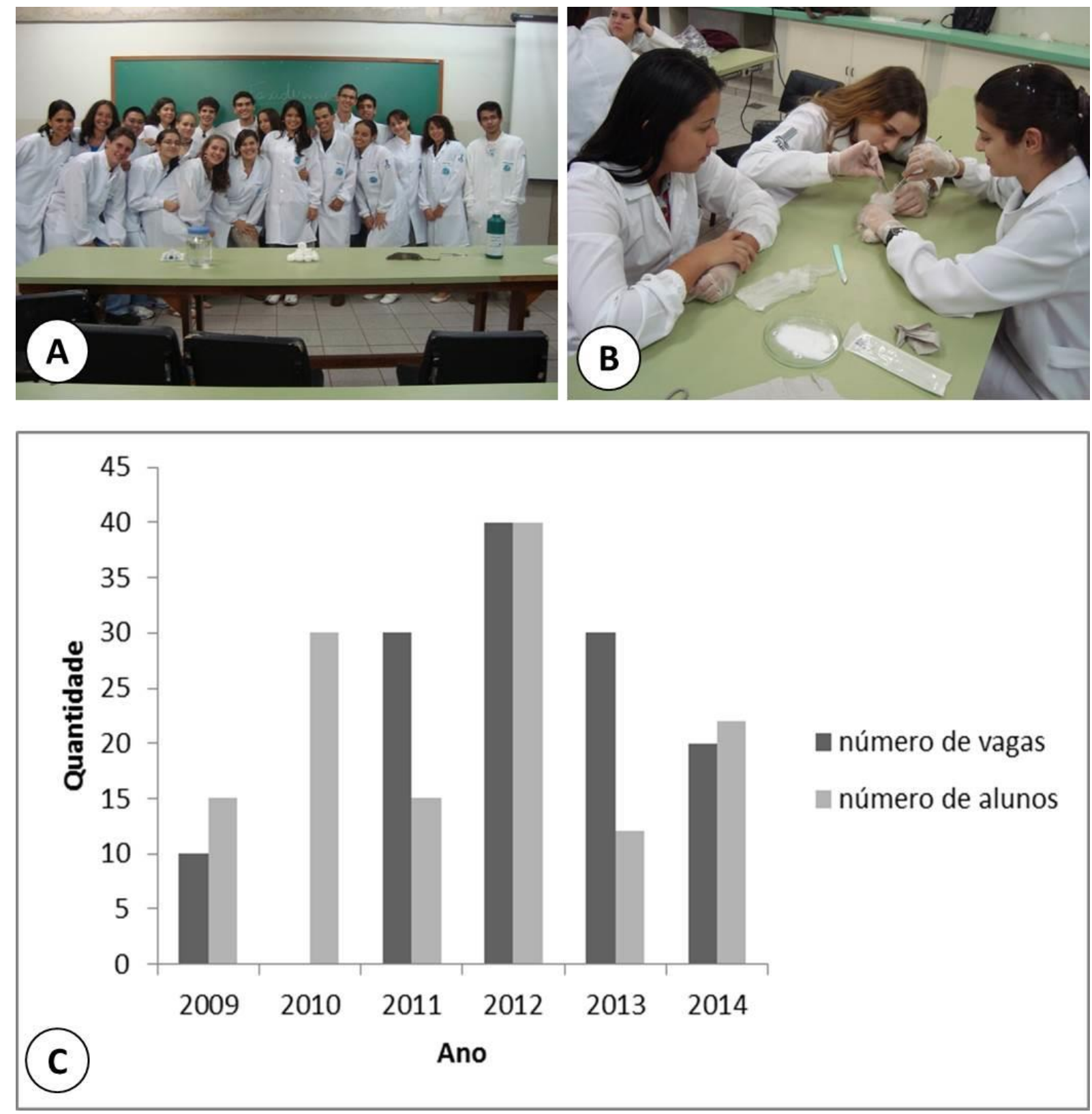

Figura 1. Cursos de Extensão em Zoologia na Universidade Federal de Mato Grosso do Sul. A. Equipe de execução e participantes do Curso Teórico Prático de Taxidermia Básica de Mamíferos (2009). B. Participantes do curso de Técnicas de Manejo de Coleções Zoológicas (2010). C. Quantidade de vagas oferecidas por ano (em 2010 não foi estabelecido número de vagas). Autoria das fotos: Thiago Mateus Rocha dos Santos.

Por fim, a terceira etapa consistiu em quatro dias e contemplou os seguintes grupos biológicos: mamíferos, aves, répteis, anfíbios e insetos. Para cada grupo foram abordados os seguintes assuntos teóricos: caracterização biológica (evolução, principais características e 
Cursos de extensão em zoologia e suas implicações no ambiente acadêmico

identificação); métodos de captura e manipulação; adequação de métodos para trabalhos científicos; métodos de morte; e conservação da coleção zoológica. Após a abordagem teórica, a parte prática foi desenvolvida com auxílio dos ministrantes e monitores aplicando, sempre que possível, a teoria ministrada a. Os participantes capturaram ou simularam a captura de animais, sendo então aplicados os conceitos de manipulação e morte.

O segundo curso contou com 30 vagas e sua divulgação abrangeu as comunidades interna e externa à UFMS, contando com cartazes de divulgação, processo adotado nas demais edições. A ampla procura pelo curso aliada a flexibilidade do edital permitiu o aceite de mais cinco participantes, totalizando 35 contemplados. O público deste curso foi constituído por estudantes de graduação em Ciências Biológicas da UFMS e da UCDB (Universidade Católica Dom Bosco). A carga horária de 40 horas permitiu o contato dos participantes com várias subáreas da Zoologia, como morfologia, sistemática e evolução. Houve apenas uma desistência, porém os demais participantes completaram a carga horária mínima exigida (75\%). Sob o ponto de vista avaliativo, novamente a maioria dos participantes considerou a proposta como "ótima". Diante deste quadro, concluímos que esta ação também foi significante e atingiu os objetivos de forma esperada, criando subsídios para as propostas elaboradas nos anos seguintes.

Embora os cursos tenham abordado conteúdos similares (técnicas para estudo zoológico), o segundo tinha como objetivo principal capacitar futuros profissionais e despertar o interesse em atividades de manejo e elaboração de coleções zoológicas. Certamente, este foi o diferencial do curso, tendo em vista a carência de profissionais que se dedicam a esta atividade atualmente.

Após o primeiro curso “Técnicas e Manejo de Coleções Zoológicas”, mais quatro ações de extensão foram desenvolvidas, sendo a última ocorrida em 2014. Nos anos de 2011 e 2013, a quantidade de participantes foi menor que a disponibilidade de vagas (Figura 1C), cenário oposto às primeiras edições. Atribuímos este número a deficiências na divulgação e ao período de realização dos cursos, o qual implicou na sobreposição com outras atividades acadêmicas e, portanto, comprometeu a disponibilidade dos potenciais participantes.

\section{CONSIDERAÇÕES FINAIS}

Anteriormente ao início da realização destes cursos de extensão havia grande demanda por conhecimento técnico na área de Zoologia na Universidade Federal de Mato Grosso do Sul. 
Cursos de extensão em zoologia e suas implicações no ambiente acadêmico

Esse conhecimento geralmente não era suprido pelas aulas de graduação (ou pós-graduação), visto que muitas vezes eram superficiais, sobretudo necessitavam de exemplos práticos para uma compreensão efetiva.

Além do conhecimento científico, entender as relações entre os animais faz parte da curiosidade de todos. Qualquer pessoa é um pouco zoóloga, classificando, mesmo que popularmente, nossa diversidade faunística. Biólogos e estudantes de Biologia vão mais longe e querem compreender as relações científicas entre os grupos. Embora tal processo não seja rápido, tão pouco fácil, o oferecimento de cursos e outras abordagens que visem subsidiar este conhecimento é um grande passo na construção de novos profissionais para atender a demanda do mercado de trabalho.

Com o início das atividades de extensão em Zoologia é clara a mudança significativa no desenvolvimento da área na UFMS. Diversos acadêmicos utilizaram o curso como uma ferramenta inicial, muitas vezes se estabelecendo posteriormente em estágios e pós-graduações. A abertura de um curso de Pós-Graduação em Biologia Animal também contribuiu para o desenvolvimento da Zoologia no âmbito da UFMS, promovendo a inserção do acadêmico no ambiente científico.

Ambos os cursos atingiram seus objetivos, bem como a aceitação da abordagem utilizada, fatores que impulsionaram a continuidade do projeto e subsidiaram o desenvolvimento de novas propostas. A aproximação entre a Universidade e a comunidade colabora para a educação de qualidade (MANCHUR et al. 2013), assim, profissionais e a população em geral poderão compreender cada vez mais os animais e deter conhecimentos básicos para aplicá-los em seu diaa-dia.

\section{REFERÊNCIAS}

BORGES, G. L. A. Formação de Professores de Biologia, Material Didático e Conhecimento Escolar. 2000. 436 p. Tese (Doutorado em Educação) - Faculdade de Educação, Universidade Estadual de Campinas, Campinas, 2000.

BRUSCA, R. C. e BRUSCA, G. J. Invertebrados. 2. ed. Guanabara Koogan, p. 968, 2007. 
Cursos de extensão em zoologia e suas implicações no ambiente acadêmico

DIAMOND, J. Evolution, consequences and future of plant and animal domestication.Nature, vol. 418, p.700-707, 2002.

GOLDENBERG, S. Aspectos éticos da pesquisa com animais. Acta Cirurgica Brasileira, vol.15, n.4, 2000.

GUIMARÃES, M. A. Uma proposta de ensino de zoologia baseada na sistemática filogenética. In: XII Encontro Nacional de Didática e Prática de Ensino- Conhecimento local e conhecimento global, 2004. Anais do XII ENDIPE Conhecimento local e conhecimento global Curitiba, p. 1074-1084, 2004.

MARQUES, Lo-Huama Santos. Universidade dinamiza o ensino de zoologia nas escolas. Estendendo Extensão, Juiz de Fora, 02 maio 2011. Disponível em: <http://www.ufjf.br/proex/files/2011/05/Maio2011.pdf> Acesso em: 01 fevereiro 2012.

MORAN, M. J. et al. Novas Tecnologias e Mediações Pedagógicas. 10. ed. Editora Papirus, Campinas, p. 173, 2006.

MANCHUR, J. et al. A contribuição de projetos de extensão na formação de profissional de graduandos de licenciatura. Revista Conexão UEPG, vol. 9, n.2, p. 334-341, 2003.

PALAUS, J. La Taxidermia. 1. ed. Editorial de Vecchi, Barcelona, p. 142,1977.

RAMOS, T. J. e CARNEIRO, G. K. T. Novas Tecnológias na Escola. Revista Digital Publicar, vol.1, n.2, p.70-88, 2009.

SCHNAIDER, T. B. e SOUZA, C. Aspectos éticos da experimentação animal. RevistaBrasileira de Anestesiologia, vol.53, n.2, p. 278-285, 2003.

VIGNE, JD. The origins of animal domestication and husbandry: A major change in the history of humanity and the biosphere. ComptesRendusBiologies, vol. 334, p. 171-181, 2011. 